\title{
Rate dependency of depth in nanoindentation of polycrystalline NiTi
}

\author{
A. Amini, Q.P. Sun * \\ Department of Mechanical Engineering, the Hong Kong University of Science and Technology, \\ Clear Water Bay, Kowloon, Hong Kong SAR, China
}

\begin{abstract}
The recent increased use of shape memory alloys (SMAs) for engineering applications manifests the need of checking the aspect of rate in NiTi. The ability of models and experiments to accurately predict the rate dependency of function-rate relationship is important. This paper concentrates on the rate dependency of depth in nanoindentation of NiTi where different tips have been used. To explain the phenomena, hysteresis damping areas are investigated. The results show decreasing depth at higher rates is due to the amount of latent heat generated from phase transition and relaxation time for heat release.
\end{abstract}

\section{INTRODUCTION}

Shape memory alloys (SMA) have been found to be interesting smart nano-composite materials including mostly $\mathrm{Ni}$ and $\mathrm{Ti}$ as they have anomalous wear properties and recoverable property. These materials are sensitive to temperature and loading rate. Although these materials, at high rates, behave like steel, at lower ones where the absolute amount of the rate is less than double time of the maximum load, they show a high sensitivity to the rate in variables. Moreover, prior experimental data indicate that a strong rate-dependency of superelastic NiTi exists in its typical properties such as variation of hysteretic shape, transformation plateau, energy dissipation, depth and temperature at different loading rates which are critical to the performance of SMA. But the rate effect of indentation for NiTi has not been considered till date. [1], [2] This paper describes experiments on the rate effect in NiTi and the results.

The dependency of depth on the rate is due to the lattice reaction in phase transition and heat transfer inside the body of the material or with the ambient. The stress-induced martensite bands on the surface of these materials and also changing wear resistance which depends on the temperature start point of martensite transformation, Ni atomic percentage, and recovery, causes strange and interesting plastic deformation recovery and damping property, and hence make them suitable as material for medical tools and even superficial muscles, joint and exterior throat layer and could also be used in the future for the wall of stomach layer. Moreover, NiTi will be useful due to the morphology change on their surface by different heating conditions and loading rates for internal piston surface in cars to improve the combustion flow and make the car engine more efficient. The rate effect in NiTi can be seen in the extensive range of 5 order magnitude in rate. This property can be exploited in the slow phenomena such as fatigue and bio tissue growth in porous NiTi to the quick damper in bearing and so on. [3], [4] There have been few theoretical derivations and experiments to measure the rate effect in the indentation of NiTi.

\footnotetext{
${ }^{*}$ Corresponding author. Tel.: +852 2358 8655; fax: +8522358 1543.

E-mail address: meqpsun@ust.hk (Q.P. Sun).
}

This is an Open Access article distributed under the terms of the Creative Commons Attribution-Noncommercial License 3.0, which permits unrestricted use, distribution, and reproduction in any noncommercial medium, provided the original work is properly cited. 
Analysis has shown that in the case of dynamic loading, the thermal effects due to the hysteresis loop and transformation latent heat cannot be neglected because they have a great influence on the alloy behavior. This property is caused by close coupling of the mechanical and thermal effects. Furthermore, the hysteresis loop is strongly conditioned by strain rate values, and must be accounted for in the case of applications.

\section{EXPERIMENTS AND DISCUSSION}

To show the rate dependency, some experiments were conducted. Table 1 shows the NiTi phase transition temperatures. These temperatures includes $M_{s}$ (martensite phase start), $M_{f}$ (martensite phase finish), $R_{s}$ (R-phase start), $R_{f}$ (R-phase finish), and $A_{s}$ (austenite phase start) and $A_{f}$ (austenite phase finish).

\begin{tabular}{|ccccccc|} 
phase & $M_{s}$ & $M_{f}$ & $R_{s}$ & $R_{f}$ & $A_{s}$ & $A_{f}$ \\
$\mathrm{SME}{ }^{\circ \mathrm{C}}$ & $\mathbf{- 1 0}$ & $-\mathbf{- 4}$ & $\mathbf{4 7}$ & $\mathbf{3 8}$ & $\mathbf{4 0}$ & $\mathbf{5 0}$ \\
\hline $\mathrm{SE}^{{ }^{\circ} \mathrm{C}}$ & $<-40$ & $<<-40$ & $\mathbf{3 0}$ & $\mathbf{1 2}$ & $\mathbf{1 0}$ & $\mathbf{3 2}$ \\
\hline
\end{tabular}

Table 1. Phase transition temperatures for polycrystalline NiTi pseudoelastic (SE) and Shape memory effect (SME)

Figure 1 shows the rotating of the curves in indentation by increasing the loading rate. The material is super elastic NiTi and the experiment temperature is $23{ }^{\circ} \mathrm{C}$; also, the tip is spherical and the maximum load is fixed at $1000 \mu \mathrm{N}$. The loading rate changes from 100 to $700 \mu \mathrm{N} / \mathrm{sec}$. These conditions help to check the rate dependency on NiTi behavior in nanoindentation depth and micro load. The roughness of the experimented material is less than 6 nanometers where the size effect is quite small. By checking the indentation curve in this chart, it is clear that by increasing the rate, the curve inclined to a lower depth and also larger hysteresis area. This rotation also involves more recovery due to the lower depth. It is seen that the rotation is limited at high loading rates. Moreover, loaddepth indentation curves almost coincide with each other due to the poor heat transfer inside the material at high loading rates.

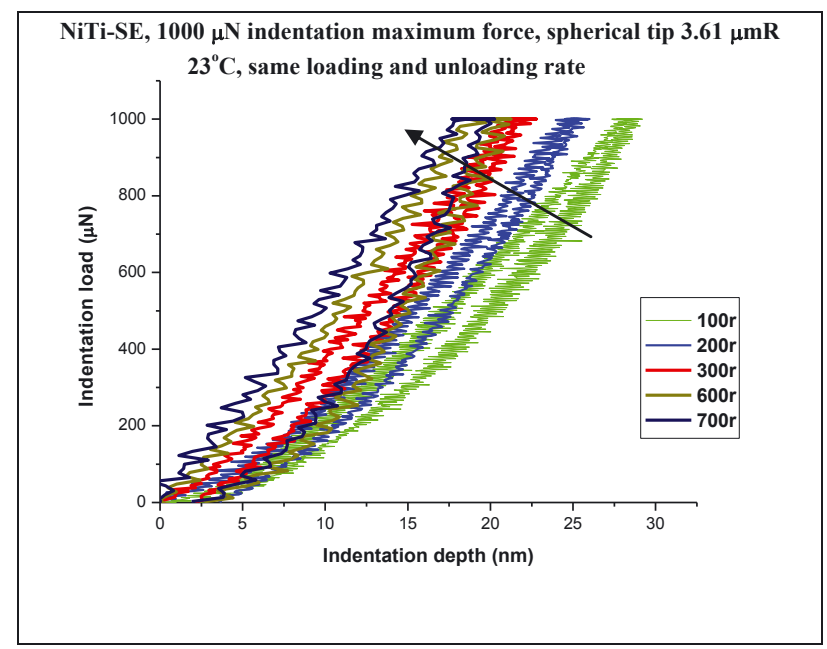

Fig. 1. Indentation load versus depth at different loading rates 
To show the rate dependency of depth in NiTi in different phases, indentations have been conducted in temperatures from $-7{ }^{\circ} \mathrm{C}$ to $135^{\circ} \mathrm{C}$ and the results are shown in figure 4 . In this figure, by increasing temperature, depth dependency decreases.
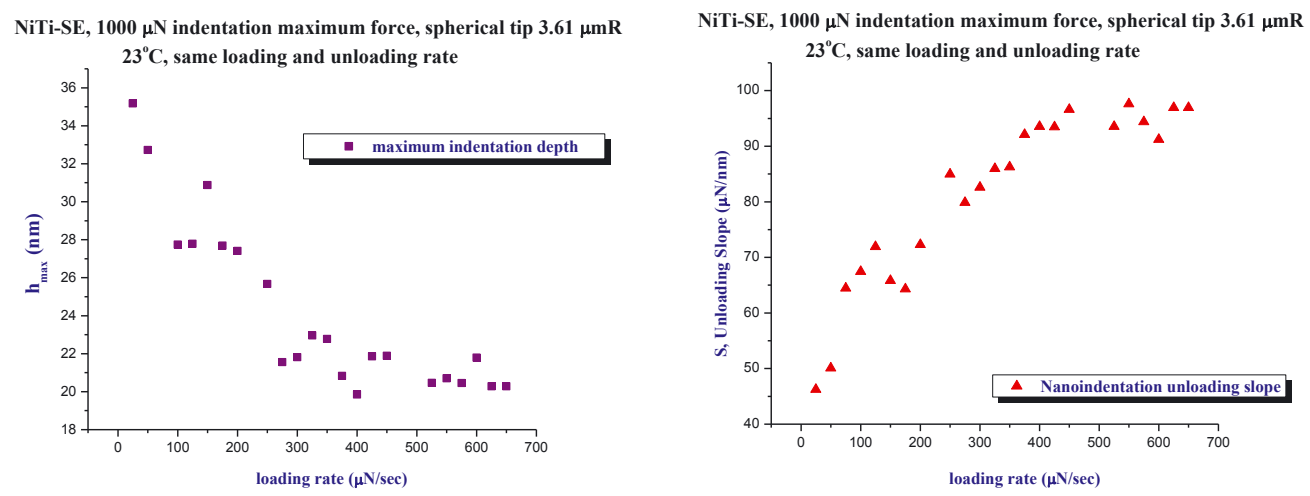

Fig. 2. Indentation depth and unloading slope versus loading rate

Maximum indentation depth and unloading slope are important criteria for the widely used method of OliverPharr in indentation. Changing these criteria causes to gain different material properties in output from Oliver-Pharr method (Hardness, $\sim 40$ percent, Modulus Elasticity, 65 percent) in NiTi at different rates. (Figure 3) These differences are ignored in industries.
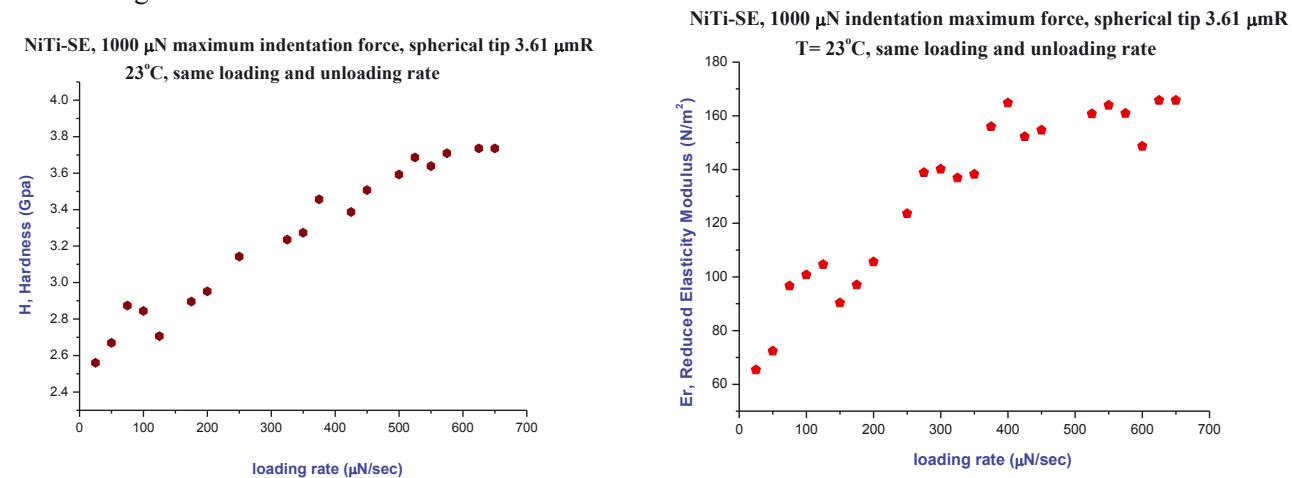

Fig. 3. Material properties from the Oliver-Pharr method, $E_{r}=\frac{\sqrt{\pi}}{2 \sqrt{A\left(\alpha h_{\max }\right)}} S, H=\frac{P_{\max }}{A\left(\alpha h_{\max }\right)}$

Also, the hysteresis area decreases by increasing the temperature. Moreover, at higher temperatures the loading rate effect disappears. In figure $5, \sigma_{t}$ (phase transition stress) increases linearly with temperature, so, at higher temperatures, the amount of transformed volume from austenite to martensite lowers because the stress does not reach the level of transformation, except right underneath the indenter where the stress is high. As a result, at higher temperatures, the area of hysteresis which represents phase transition decreases. A lower amount of transformed volume (or the sum of the released latent heat and its rate of conduction or convection from the indentation area) and lower rate dependency verify that the source of dependency lies in the phase transition in NiTi. To explain the phenomena in indentation, figure 6 illustrates the stress induced latent heat of phase transition from austenite to martensite underneath the spherical indenter. This heat is transferred by conduction through the body of NiTi to the adjacent area and also from the contacted tip area in addition to convection to the environment. At higher rates one 
can see two phenomena, firstly, latent heat does not have a chance to be released on time and it accumulates, and secondly, the phase transition stress level increases by increasing the temperature in this zone. (Figure 3-5)

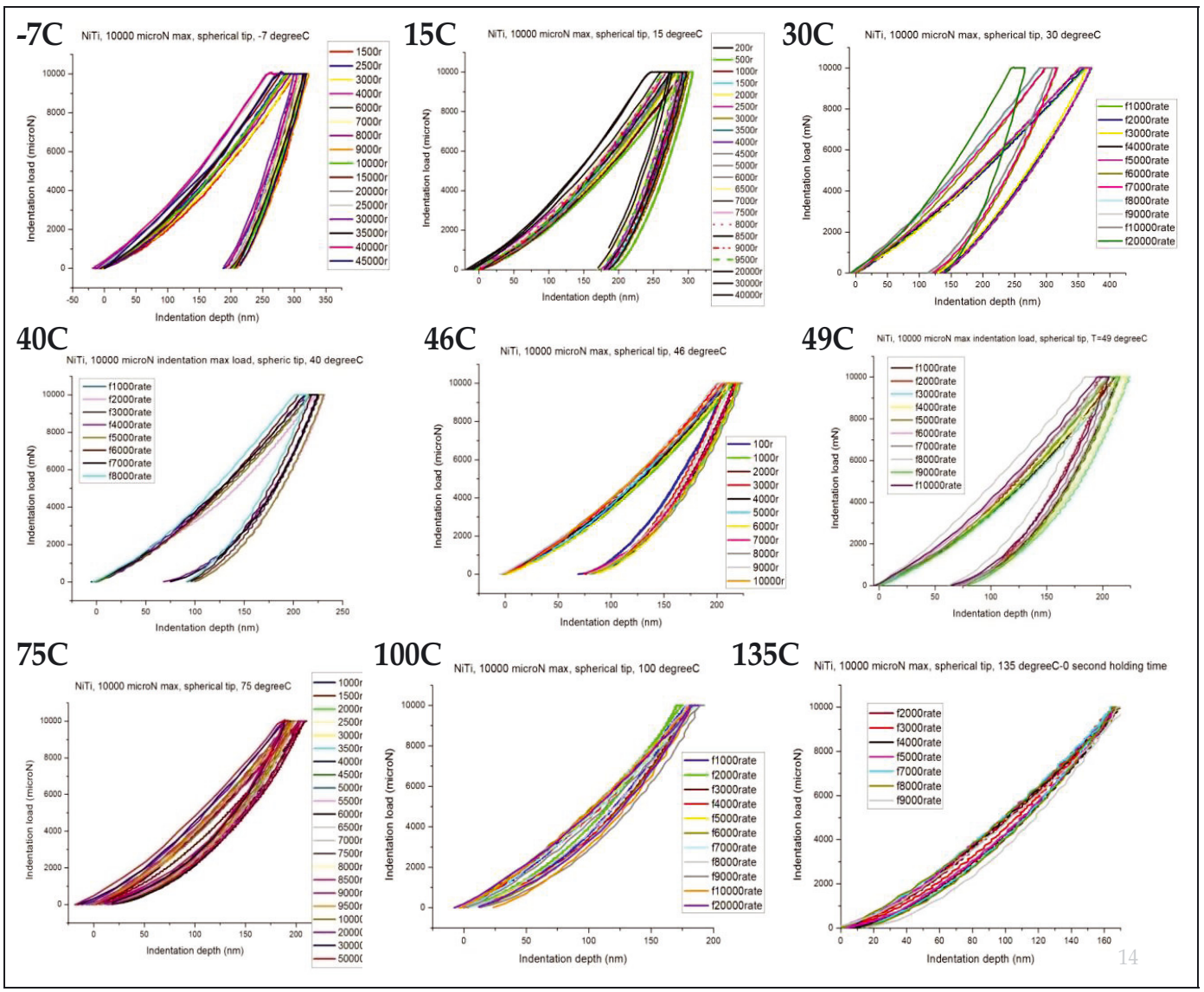

Fig. 4. Maximum indentation depth dependency on loading rate for different temperatures

At higher temperatures, the gradient of the conducted heat lessens, this helps the accumulation of heat, raising the temperature and postponing the phase transition. However, right underneath the tip, the stress is high enough to catch the transition stress, so, in $135{ }^{\circ} \mathrm{C}$ a hysteresis area exists although rate dependency disappears. In figure 3 , at higher rates, saturation happens. This saturation means that at higher rate depth does not depend on rate anymore. This is from the fact that at high rates, NiTi behaves similar to ordinary materials. In other words, a mechanical load is applied to the materials and due to the high level of phase transition stress, phase transition does not occur. The same process happens in cyclical loads. Figure 7 demonstrates 30 cyclic loading with a fixed maximum load of $10000 \mu N$ and nanoindentation applied at the same point. After a few cycles the temperature of the indentation zone increases by accumulation of latent heat. In the end, the sensitivity of the depth decrease disappears and curves are saturated. Due to the larger amount of phase transition volume with the condition of lower temperature and lower transition stress, the hysteresis area of the first cycle is larger than that of the last cycle. On the contrary, ordinary materials such as quartz (Silica), which is standard material for indentation calibration, do not show rate dependency of indentation depth. Figure 8 shows the result for quartz at different loading rates. In this figure there are no depth and hysteresis changes at different rates. Under initial condition, SME (Shape Memory Effect) NiTi was kept 
in $-40^{\circ} \mathrm{C}$ for two hours and then cooled down to room temperature (testing temperature) gradually. This causes twinned microstructure in martensite, as SE is in a twinned R-phase condition (table 1, figure 10) [5], [6]. In table 2 the difference in depth change by rate change in SME is more than that in SE NiTi; also, in table 3, the released latent heat is higher in martensite, austenite and R-phase.

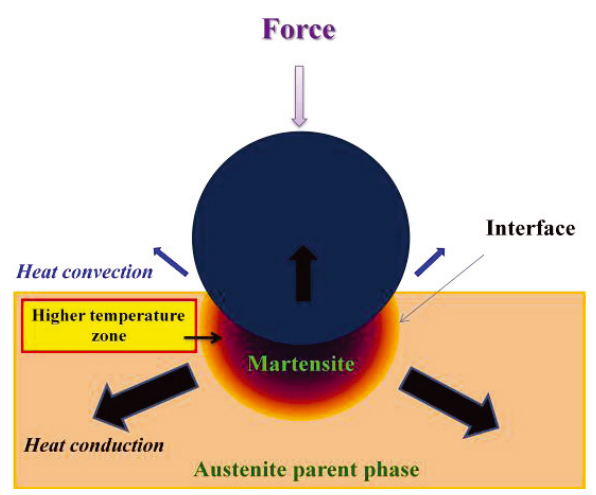

Fig. 6. NiTi indentation zone and latent heat transfer

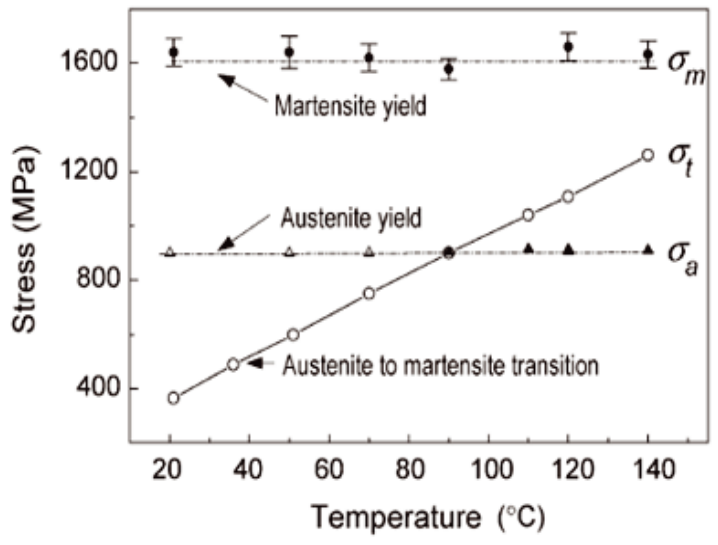

Fig. 5. Phase transition stress increases with temperature [7]

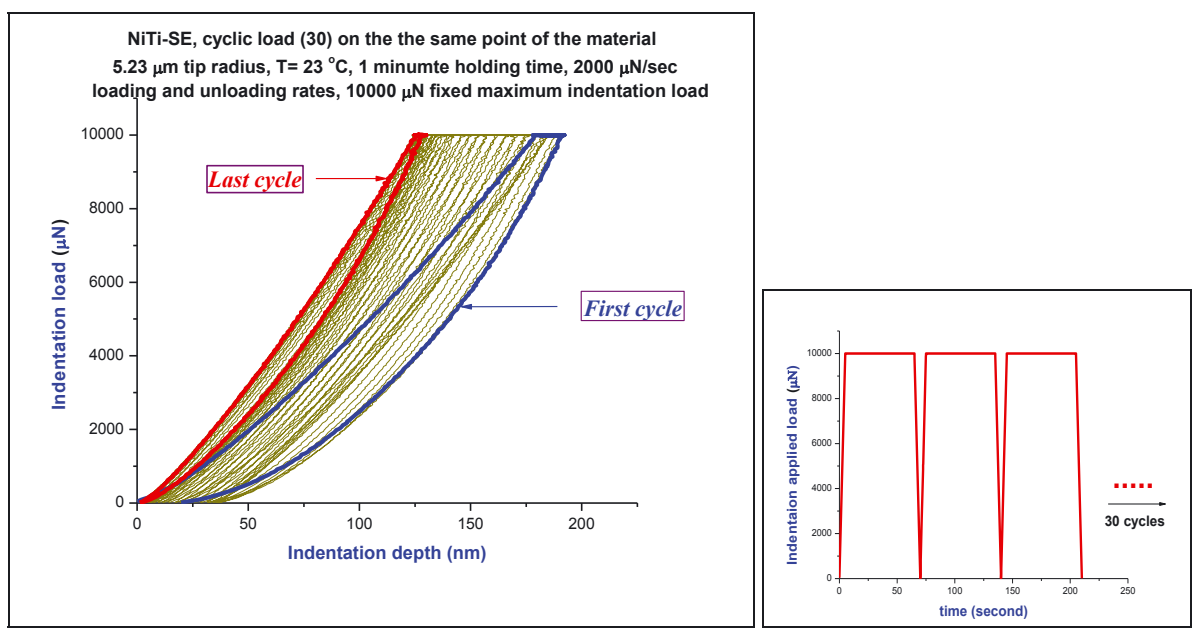

Fig. 7. Indentation results with cyclic load

By applying a compression load on NiTi, the sum-up latent heat and increase in the temperature in indentation zone in SME is more than that in SE, this causes more depth change at SME in different rates. Interestingly, complex loading and unloading rates is worth considering. This is due to the factual need of NiTi applications under different loading and unloading rates. Our experiments show that NiTi is not sensitive to a combined loading rate (hysteresis damping area). On the contrary, NiTi shows more sensitivity to the combination of the unloading rate. Figure 11 shows the results of experiments with combined rates. In these experiments, loading rates of $400 \mu \mathrm{N} / \mathrm{sec}$, and $20000 \mu \mathrm{N} / \mathrm{sec}$ is switching to each other at the half of the time of loading and unloading sectors correspondingly (loading rate (lr) and unloading rate (ulr)). Moreover, the higher loading rate shows lower indentation depth (first part in 1, and second section in 2 with $20000 \mu \mathrm{N} / \sec$ in (a)-figure 11), but, the higher 
unloading rate shows higher indentation depth (first part in 4, and second section in 3 with $20000 \mu N / \sec$ in (b)figure 11). One can expect such a behavior; this is because the higher loading rate causes that the phase transition to martensite to be postponed. Moreover, more austenite parent phase, higher hardness, higher loading slope, higher modulus elasticity with lower depth (Oliver - Pharr method). Also, higher unloading rate causes lesser

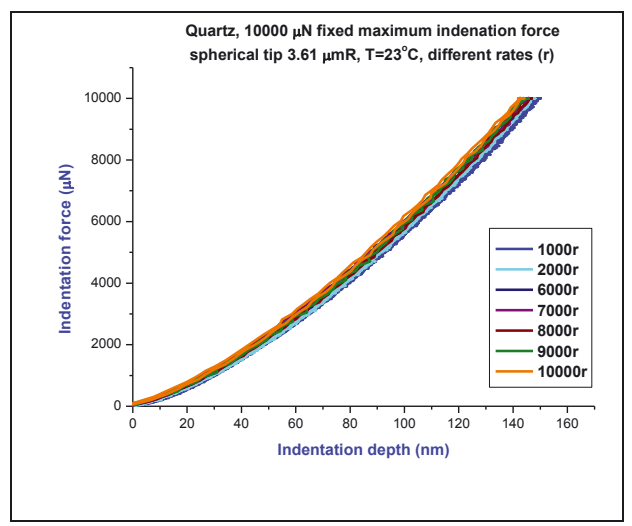

Fig. 8. Independency of indentation depth on loading rate for Quartz
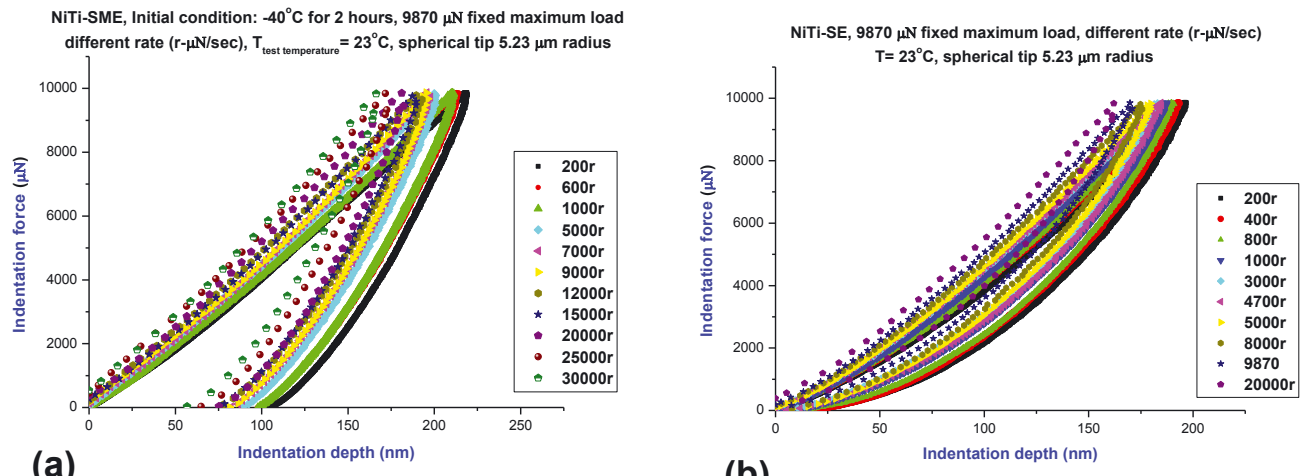

Fig. 9. SE, SME (initial condition of $-40^{\circ} \mathrm{C}$ for 2 hours)

\begin{tabular}{|l|c|c|c|}
\hline \multicolumn{1}{|c|}{$9870 \mu \mathrm{N}$ by $5.23 \mu \mathrm{mR}$ tip } & $\begin{array}{c}h_{\max }(\mathrm{nm}) \text { for } \\
200 \mu N / \mathrm{sec} \text { rate }\end{array}$ & $\begin{array}{c}h_{\max }(\mathrm{nm}) \text { for } \\
20000 \mu N / \mathrm{sec} \text { rate }\end{array}$ & $\boldsymbol{h}_{\max }(200 r)-h_{\max }(20000 r)(\mathrm{nm})$ \\
\hline SME, $-40^{\circ} \mathrm{C}$ for 2 hours & 218.52 & 181.030 & 37.49 \\
\hline SE & 196.17 & 162.09 & 34.08 \\
\hline
\end{tabular}

Table 2. Maximum depths for the condition shown in figures 9

\begin{tabular}{|c|c|c|c|c|c|}
\hline \multicolumn{3}{|c|}{ SME } & \multicolumn{3}{c|}{ SE } \\
\hline$R_{f}-R_{s}$ & $M_{f}-M_{s}$ & $A_{f}-A_{s}$ & $R_{f}-R_{s}$ & $M_{f}-M_{s}$ & $A_{f}-A_{s}$ \\
\hline$-6 \mathrm{~J} / \mathrm{gr}$ & $-8 \mathrm{~J} / \mathrm{gr}$ & $18 \mathrm{~J} / \mathrm{gr}$ & $-5 \mathrm{~J} / \mathrm{gr}$ & $-6 \mathrm{~J} / \mathrm{gr}$ & $15 \mathrm{~J} / \mathrm{gr}$ \\
\hline
\end{tabular}

Table 3. Latent heat released or absorbed per unit mass within NiTi from DSC 
cooling time, higher temperature, more remaining austenite phase, more modulus elasticity and more unloading slope. In figure 11- (c), the hysteresis area between 1 and 2 is almost 0.013 nanoJouls, although the difference between 3 and 4 is 0.046 nanoJouls (4 times higher).

It is worth mentioning that sharp tips show more rate dependency in the indentation depth than non-sharp tips (Figure 12). Table 4 shows the result of the maximum indentation depth difference for the rate of 1000 and 7000 $\mu \mathrm{N} / \mathrm{sec}$. Using a Berkovich tip, it is shown that indentation depth dependency is more sensitive to the rate in comparison to the result from spherical tip. Initiation of phase transition occurs in the areas where the concentration of stress is high and phase transition relaxes this stress concentration.

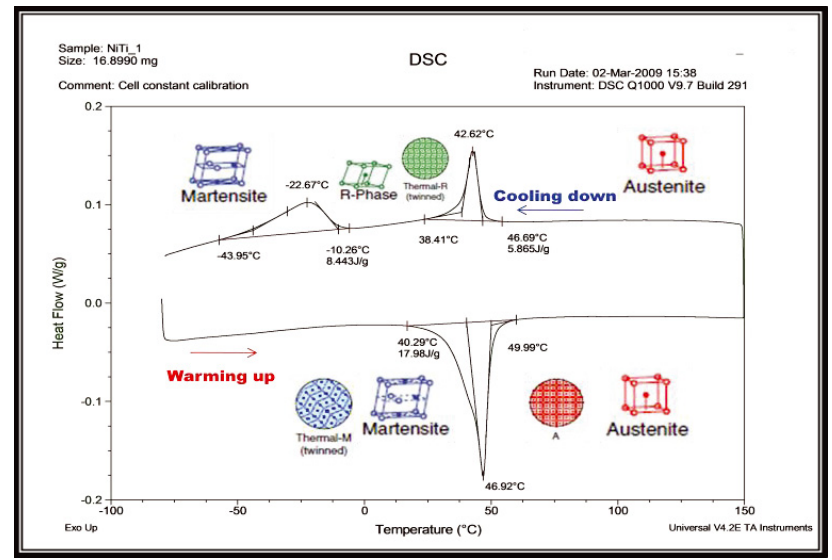

Figure 10. DSC curve for SME NiTi

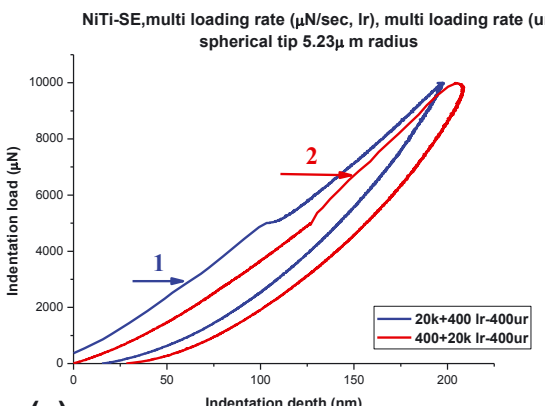

(a)

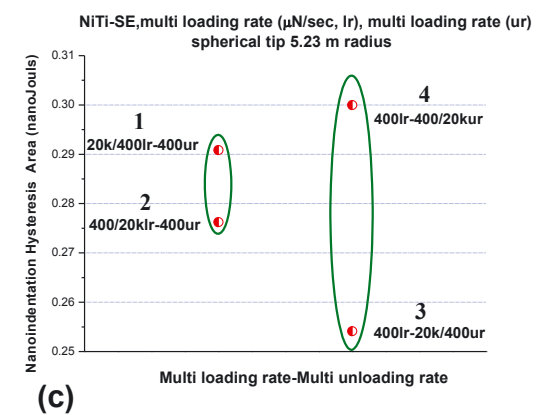

Fig. 11. Combined loading rate (Ir) and unloading rate (ulr) and hysteresis damping area

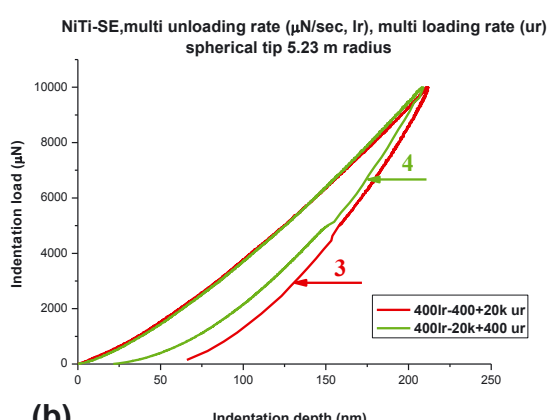

(b)

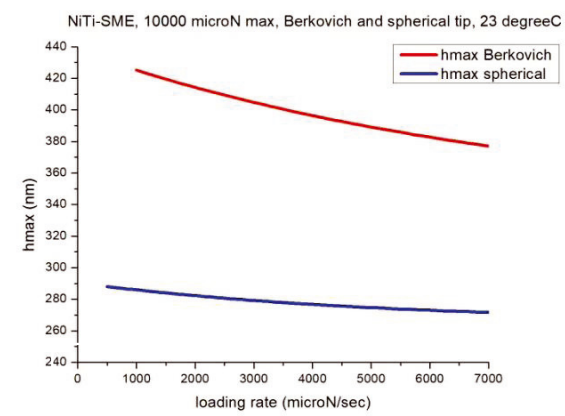

Fig. 12. More rate dependency of indentation depth by sharp tips than spherical tips 
This phenomenon happens when sharp tips are used. The Berkovich tip, compared with a spherical tip, precedes stress concentration, so the amount of phase transited volume inside the body of NiTi is higher. This causes a higher temperature in the phase transited area than in the indentation area temperature measured by spherical tip. As a result, depth shows more sensitivity (difference) to the rate in sharp tips.

\begin{tabular}{|l|c|c|c|}
\hline \multicolumn{1}{|c|}{ tips } & $\begin{array}{c}h_{\max }(\mathrm{nm}) \text { for } \\
1000 \mu N / \mathrm{sec} \text { rate }\end{array}$ & $\begin{array}{c}h_{\max }(\mathrm{nm}) \text { for } \\
7000 \mu N / \mathrm{sec} \text { rate }\end{array}$ & $\boldsymbol{h}_{\max }(1000 r)-h_{\max }(7000 r)(\mathrm{nm})$ \\
\hline Spherical tip with 3.61 $\mu \mathrm{mR}$ & 288 & 268 & 20 \\
\hline Berkovich tip & 426 & 376 & 50 \\
\hline
\end{tabular}

Table. 4. Indentation depth difference for Berkovich and spherical tips for nanoindentation with $F_{\max }=10 \mathrm{k} \mu \mathrm{N}$

\section{CONCLUSION}

The growing number of high tech applications of NiTi, especially bio-applications such as surgical tools and artificial tissues, as well as material property characterization, demands the need for rate dependency consideration of NiTi. This paper shows the rate dependency of NiTi properties through nanoindentation. It was discovered that, nanoindentation depth decreases at higher loading rates. Moreover, rate dependency depends on tip (size effect), initial condition and experiment temperature. Furthermore, it has been proven that the rate dependency of depth is due to the competition between produced latent heat amount and the time of latent heat release in comparison with the loading time. To consider this multi-scale dependency, more theoretical and experimental effort is needed.

\section{REFERENCE}

[1] Yuanxin Zhou, Wang Yang, Yuanmin Xia, P.K. Mallick, 2003, “An experimental study on the tensile behavior of a unidirectional carbon fiber reinforced aluminum composite at different strain rates," Materials science and Engineering, A362, 112-117.

[2] Dazhi Yang, 2000, "Shape memory alloy and smart hybrid composites, advanced materials for $21^{\text {st }}$ Century," Mechanical and Design, 21, 503-505.

[3] Stefan Seelecke, 2002, "Modeling the dynamic behavior of shape memory alloys," International Journal of NonLinear Mechanics 37, 1363-1374.

[4] J. Van Humbeeck, 2003, "Damping capacity of thermoelastic martensite in shape memory alloys," Journal of Alloys and Compounds, 355, 58-64.

[5] Shaw, J.A., Churchill, C.B., and Iadicola, M.A., "tips and tricks for characterizing shape memory alloy wire: part 2 - fundamental isothermal responses,' Experimental Techniques, 33 (1), pp. 51-62 (2009).

[6] Qian, L., Sun, Q., Xiao, X., "Role of phase transition in the unusual microwear behavior of superelastic NiTi shape memory alloy," Wear 260 (4-5), pp. 509-522 (2006).

[7] Shaw, J.A., Churchill, C.B., and Iadicola, M.A., "Tips and Tricks for Characterizing Shape Memory Alloy Wire: part 1-differential Scanning Calorimetry \& Basic Phenomena,' Experimental Techniques, 32 (5), pp.55-62 (2008). 